\title{
Causas e consequências da redução da cobertura vacinal no Brasil
}

\section{Causes and consequences of reducing vaccination coverage in Brazil}

Lucia Regina Nogas Milani ${ }^{1}$, Ivana Maria Saes Busato²

1. ORCID: https://orcid.org/0000-0002-3657-5426. Cirurgiã Dentista. Especialista em Saúde Coletiva. Secretaria Municipal da Saúde, Curitiba, PR, Brasil. E-mail: lunogas@ yahoo.com.br

2. ORCID: https://orcid.org/0000-0002-5643-5981. Cirurgiã Dentista. Doutora em Odontologia. Centro Universitário Internacional Uninter, Curitiba, PR, Brasil. E-mail: ivanabusato5@gmail.com

CONTATO: Autor correspondente: Lucia Regina Nogas Milani | Endereço: Rua Haiti, 81 CEP 81010-065 - Curitiba/PR. Telefone: (41) 99212-7740. E-mail: lunogas@yahoo.com.br

RESUMO A vacinação é a estratégia mais efetiva de se evitar uma doença transmissível, contudo tem-se constatado diminuição das coberturas de diversas vacinas, por razões como hesitação vacinal, esquecimento, notícias falsas sobre vacinas, entre outros. Este artigo tem como escopo elencar as principais causas e as futuras consequências na redução da cobertura vacinal, permitindo avaliar as variantes que colaborariam para um aumento no número de hesitantes, por meio de trabalho multimétodos. Avaliando-se os diferentes autores, pode-se concluir que a redução da cobertura vacinal é problemática para a saúde coletiva, causando avanço de doenças já equacionadas e o ressurgimento de doenças erradicadas, provocando danos ou morte, especialmente em crianças. Portanto, torna-se primordial por parte dos diferentes agentes de saúde, governos, trabalhadores da saúde, controle social, entidades educacionais, uma união de forças para empoderar a população, e auxiliar na desconstrução dos mitos relacionados à vacinação.

DESCRITORES: Cobertura Vacinal. Hesitação Vacinal. Imunização. 


\begin{abstract}
Vaccination is the most effective strategy to prevent a communicable disease; however, there has been a reduction in the coverage of several vaccines, for reasons such as hesitation about the vaccine, forgetfulness, fake news about vaccines, among others. This article aims to list the main causes and future consequences in the reduction of vaccination coverage, allowing the variants evaluation that would contribute to an increase in the hesitants number, through multi-method work. Assessing the different authors, it can be concluded that the reduction in vaccination coverage is a problem for public health, causing the advance of diseases that have already been considered and the resurgence of eradicated diseases, causing damage or death, especially in children. Therefore, it is essential for different health agents, governments, health workers, social control and educational entities, to join forces to empower the population, and help deconstruct the myths related to vaccination.
\end{abstract}

DESCRIPTORS: Vaccine Coverage. Vaccine Hesitation. Immunization. 


\section{INTRODUÇÃO}

O s primeiros registros de vacinação no mundo datam de 1771 na Inglaterra e tinham o objetivo de tratar a epidemia de varíola. A primeira vacina foi descoberta por Edward Jenner, em 1796, após 20 anos de estudos e experimentos com a varíola bovina, dando origem aos termos vaccine e vaccination (derivados do termo latino vacca). $\mathrm{Na}$ Inglaterra do século XVIII, a varíola era responsável por cerca de $10 \%$ dos óbitos totais e um terço das mortes era registrado entre as crianças. Classificada como uma das doenças mais devastadoras da história da humanidade, a varíola foi considerada erradicada pela Organização Mundial de Saúde (OMS) em 1980, após realização de um programa de vacinação em massa de ordem mundial ${ }^{1}$.

Particular importância é atribuída à campanha de erradicação da varíola no Brasil (1966-1973), por iniciativa do Barão de Barbacena. A experiência de vacinação antivariólica em massa da população brasileira é algo incomparável na história da saúde no Brasil, e mesmo da saúde internacional2.

O Programa Nacional de Imunizações (PNI) do Sistema Único de Saúde (SUS), criado em 1973 e coordenado pelo Ministério da Saúde, de forma compartilhada com as secretarias estaduais e municipais de saúde, é reconhecido, nacional e internacionalmente como uma das mais relevantes intervenções em saúde pública, com registro de grandes vitórias, como a erradicação da poliomielite, a eliminação da circulação do vírus autóctone da rubéola e o importante impacto na redução de doenças imunopreveníveis, contribuindo para a redução da mortalidade infantil e a melhoria da expectativa de vida da população brasileira ${ }^{3}$.

Um dos primeiros casos de hesitação vacinal no Brasil aconteceu com a "Revolta da Vacina", em 1904, na cidade do Rio de Janeiro, um movimento popular contra a vacinação obrigatória no país implementada por Oswaldo Cruz. A palavra 'hesitação' tem como um de seus significados "ato ou efeito de hesitar, estar ou ficar indeciso, ter dúvidas"4. O termo 'hesitação vacinal' é definido "como o atraso em aceitar ou a recusa das vacinas recomendadas, apesar de sua disponibilidade nos serviços de saúde"5.

O movimento antivacina é tão antigo quanto a própria vacinação. No Reino Unido, já havia caricaturas da vacina de varíola desde os anos de 1800. A obrigatoriedade da vacinação causou resistência de indivíduos que a consideravam uma invasão da liberdade sobre o próprio corpo. Nos Estados Unidos, brigas judiciais contra a vacinação obrigatória não eram raras nos anos $1920^{6}$.

Afinal, por que têm ocorrido reduções tão importantes na cobertura vacinal brasileira? De que forma os movimentos antivacina e a hesitação vacinal têm colaborado para tal decréscimo? O Estado e a Administração Pública podem intervir de que maneira 
para que tal situação seja controlada? Tendo estas reflexões, objetivou-se descrever o fenômeno da redução da cobertura vacinal no território nacional, por meio da verificação de suas causas e consequências, além de demonstrar ações implementadas pela Administração Pública de forma a manter as coberturas vacinais preconizadas pelo Ministério da Saúde.

\section{MÉTODO}

Trata-se de um trabalho multimétodos, um estudo qualitativo realizado por meio da obtenção de dados epidemiológicos e publicações que abordam o tema, por meio de ampla revisão bibliográfica. As principais informações estão baseadas nos fundamentos e objetivos de ação do Programa Nacional de Imunizações do Ministério da Saúde. Os dados epidemiológicos são descritos com base nos boletins de acesso público divulgados pelas Secretarias de Saúde municipais e estaduais. Realizou-se também uma revisão integrativa sobre artigos relacionados ao tema dos movimentos antivacinas, em sites de busca PubMed, Google Scholar, SciELO, Science Direct utilizando-se os seguintes descritores: hesitação vacinal, cobertura vacinal, movimentos antivacinas, fake news, selecionando-se artigos publicados entre 2015 a 2019 nos idiomas português e inglês. Foram realizados cruzamentos entre as palavras-chave, utilizando-se os seguintes critérios: "Hesitação Vacinal" and "Cobertura Vacinal" e "Movimentos antivacinas" and "fake news".

\section{RESULTADOS}

A análise dos dados epidemiológicos, com base nos boletins de acesso público divulgados pelas Secretarias de Saúde municipais e estaduais, mostrou que há um decréscimo das coberturas vacinais, os documentos não relacionam diretamente a hesitação vacinal ou movimentos antivacinas para essa ocorrência. O resultado da revisão integrativa, após avaliação dos artigos, utilizando as palavras-chave, resultou num total de sete artigos que efetivamente apresentaram informações para responder às perguntas desta pesquisa: motivos da redução na cobertura vacinal, relação entre movimentos antivacina e hesitação vacinal e a atuação do Estado e da Administração Pública nestes quesitos, foram critérios de inclusão nesse estudo. Os artigos estudados variam entre os anos de 2015 a 2019, foi constatado que existem poucos estudos que relatam as causas da hesitação vacinal. Os artigos desta revisão são apresentados no Quadro 1. 
Quadro 1. Resultados da revisão integrativa, 2020.

\begin{tabular}{|c|c|c|}
\hline Autores e data & Título do artigo & Objetivo do artigo \\
\hline SATO $(2018)^{5}$ & $\begin{array}{l}\text { Qual a importância da } \\
\text { hesitação vacinal na } \\
\text { queda das coberturas } \\
\text { vacinais no Brasil? }^{5}\end{array}$ & $\begin{array}{l}\text { Apresentar a definição e os fatores } \\
\text { relacionados à hesitação vacinal, } \\
\text { bem como discutir sua importância no } \\
\text { contexto brasileiro. }\end{array}$ \\
\hline MACDONALD $(2015)^{12}$ & $\begin{array}{l}\text { Vaccine hesitancy } \\
\text { definition, scope and } \\
\text { determinants }^{12}\end{array}$ & $\begin{array}{l}\text { Através do estudo do escopo e dos } \\
\text { seus determinantes, padronizar } \\
\text { definição para "hesitação vacinal". }\end{array}$ \\
\hline JARRETT et al. (2015) $)^{19}$ & $\begin{array}{l}\text { Strategies for } \\
\text { addressing vaccine } \\
\text { hesitancy - a } \\
\text { systematic review }{ }^{19}\end{array}$ & $\begin{array}{l}\text { Identificar, descrever e avaliar a eficácia } \\
\text { potencial de estratégias para responder } \\
\text { a questões de hesitação vacinal que } \\
\text { foram implementadas e avaliadas em } \\
\text { contextos globais diversos. }\end{array}$ \\
\hline DUBÉ $(2017)^{23}$ & $\begin{array}{l}\text { Addressing vaccine } \\
\text { hesitancy: the crucial } \\
\text { role of healthcare } \\
\text { providers } \\
23\end{array}$ & $\begin{array}{l}\text { Apresentar as conclusões de um estudo } \\
\text { conduzido entre médicos clínicos gerais } \\
\text { na França sobre as discrepâncias } \\
\text { entre as recomendações de vacinas e } \\
\text { atualizações da carteira vacinal para os } \\
\text { pacientes e para seus familiares. }\end{array}$ \\
\hline $\begin{array}{l}\text { AVAAZ; SOCIEDADE } \\
\text { BRASILEIRADE } \\
\text { IMUNIZAÇÕES (SBIm) } \\
(2019)^{9}\end{array}$ & $\begin{array}{l}\text { As fake news estão } \\
\text { nos deixando } \\
\text { doentes? }{ }^{9}\end{array}$ & $\begin{array}{l}\text { Descrever como a desinformação } \\
\text { antivacinas pode estar reduzindo as } \\
\text { taxas de cobertura vacinal no Brasil. }\end{array}$ \\
\hline MCCLURE et al. $(2017)^{21}$ & $\begin{array}{l}\text { Vaccine Hesitancy: } \\
\text { Where We Are and } \\
\text { Where We Are } \\
\text { Going }^{21}\end{array}$ & $\begin{array}{l}\text { O artigo foca na hesitação e recusa das } \\
\text { vacinas preconizadas pelo Calendário } \\
\text { Vacinal infantil nos Estados Unidos, } \\
\text { ainda que muitos dos conceitos } \\
\text { discutidos possam ser aplicados } \\
\text { em outros países e na vacinação } \\
\text { dos adultos, e nas estratégias de } \\
\text { abordagem sobre este assunto. }\end{array}$ \\
\hline FERREIRA et al. $(2019)^{24}$ & $\begin{array}{l}\text { Counteracting the } \\
\text { contemporaneous } \\
\text { proliferation of digital } \\
\text { forgeries and fake } \\
\text { news }\end{array}$ & $\begin{array}{l}\text { Apresentar métodos aplicáveis à } \\
\text { verificação de conteúdo duvidoso em } \\
\text { texto e imagens e discutir como colocá- } \\
\text { los juntos como uma opção para conter } \\
\text { a proliferação de "fatos" não verificados } \\
\text { e falsos. }\end{array}$ \\
\hline
\end{tabular}

Fonte: Elaborado pelas autoras (2020). 


\section{DISCUSSÃo}

A vacinação é uma das maneiras mais efetivas de se evitar doenças. Atualmente previne 2-3 milhões de mortes ao ano, e mais de 1,5 milhão poderiam ser evitadas se a cobertura vacinal mundial fosse aprimorada. No entanto, a hesitação vacinal está entre as 10 ameaças à saúde mundial. Juntamente com resistência antimicrobiana, HIV e dengue, a resistência ou recusa à vacinação tem sido uma questão global crescente ${ }^{7}$.

O Brasil é um dos países que oferece o maior número de vacinas à população. Atualmente, o Programa Nacional de Imunizações, PNI, disponibiliza mais de 300 milhões de doses anuais distribuídas entre 44 imunobiológicos, incluindo vacinas, soros e imunoglobulinas. Conta com aproximadamente 34 mil salas de vacinação e 42 Centros de Referência em Imunobiológicos Especiais (CRIE), que atendem indivíduos portadores de condições clínicas especiais e utilizam variadas estratégias de vacinação, incluindo vacinação de rotina, campanhas, bloqueios vacinais e ações extramuros ${ }^{8}$.

O ressurgimento de doenças como o sarampo em nações onde este já era erradicado é só um dos exemplos de como a hesitação vacinal pode ser um sinal de ameaça à saúde pública. Os prejuízos causados pela falta de vacinação já estão se tornando realidade, tendo como exemplo o sarampo que apresenta mais de 300 casos confirmados em Roraima e no Amazonas 5 . Ainda, segundo dados do próprio PNI, nos últimos dois anos as coberturas vacinais entre menores de 1 ano de idade foram inferiores ao mínimo desejado. O período crítico começou em 2017, quando $25 \%$ da população em risco de contrair febre amarela, a maioria crianças, não foi vacinado. O problema permanece crítico: dados recentes revelam que apenas $88 \%$ da população-alvo foi vacinada contra sarampo, em todo o país este ano (o percentual mínimo para a eliminação desta doença é 95\%), enquanto em 100 municípios brasileiros, a cobertura alcançou menos de $50 \%$ da população-alvo contra a poliomielite ${ }^{9}$.

No Brasil, a redução da cobertura vacinal é preocupante, de acordo com dados do Ministério da Saúde ${ }^{10}$, como se verifica no Quadro 2.

Quadro 2. Proporção de municípios com coberturas vacinais adequadas por tipo de vacinas (Homogeneidade de coberturas vacinais), Brasil. 2011 a 2016.

\begin{tabular}{|l|r|r|r|r|r|r|}
\hline Imunobiológicos & $\mathbf{2 0 1 1}$ & $\mathbf{2 0 1 2}$ & $\mathbf{2 0 1 3}$ & $\mathbf{2 0 1 4}$ & $\mathbf{2 0 1 5}$ & $\mathbf{2 0 1 6}$ \\
\hline BCG & 53,7 & 47,4 & 40,1 & 46,2 & 54,9 & 44,5 \\
\hline Poliomielite & 71,2 & 57,5 & 44,7 & 51,2 & 60,4 & 43,1 \\
\hline DTP/HiB/HB & 70,4 & 54,8 & 59,9 & 49,7 & 64,0 & 50,5 \\
\hline Rotavírus & 58,0 & 52,7 & 44,7 & 50,6 & 71,0 & 59,9 \\
\hline Pneumocócica & 47,0 & 49,3 & 56,8 & 48,8 & 60,7 & 59,5 \\
\hline Meningococo C & 72,4 & 52,2 & 64,1 & 50,0 & 65,5 & 54,3 \\
\hline Tríplice Viral & 65,0 & 61,4 & 75,1 & 55,2 & 58,8 & 58,9 \\
\hline
\end{tabular}

Fonte: MS/SVS/DEVIT/CGPNI/Sistema de Informação do Programa Nacional de Imunizações (http://pni.dstasus.gov.br). 
A cobertura vacinal adequada é fator primordial para a proteção da população nas diversas faixas etárias, pois tem o poder de impedir a proliferação de determinadas doenças e/ou até mesmo erradicá-las, impactando positivamente a saúde pública.

Mais da metade das cidades brasileiras não têm cobertura adequada para a maioria das vacinas do calendário nacional. Apenas $44,6 \%$ dos municípios alcançaram a meta estipulada para a pólio, algo semelhante ocorrendo com hepatite A, BCG (que previne contra formas graves da tuberculose), rotavírus, meningocócica $\mathrm{C}$ e pentavalente. Em junho de 2018, o órgão fez um alerta de que 312 municípios haviam vacinado menos de $50 \%$ das crianças na faixa etária recomendada contra a pólio. A queda na cobertura também é percebida nos estados: o Rio Grande do Norte, por exemplo, vacinou apenas cerca de $68 \%$ da população pretendida contra a doença, em 2017 — enquanto antes de 2015, o estado sempre havia alcançado cobertura acima de $90 \%{ }^{11}$.

Quando se analisa o panorama de Curitiba nos últimos seis anos (Figura 1), verifica-se um panorama relativamente estável, excetuando-se a vacina pentavalente, a qual teve desabastecimento no ano de 2019, o que supostamente haveria causado a redução da imunização por esta vacina.

Figura 1. Série Histórica da Cobertura Vacinal - Curitiba. 2014 a 2019.

\begin{tabular}{|c|c|c|c|c|c|c|}
\hline & 2014 & 2015 & 2016 & 2017 & 2018 & $1019^{*}$ \\
\hline BCG & $87,7 \%$ & $99,5 \%$ & $93,6 \%$ & $98,6 \%$ & $96,4 \%$ & $98,9 \%$ \\
\hline ROTAVÍRUS & $92,9 \%$ & $86,4 \%$ & $84,6 \%$ & $86,2 \%$ & $90,3 \%$ & $90,9 \%$ \\
\hline MENINGO C & $95,4 \%$ & $97,9 \%$ & $93,5 \%$ & $32,0 \%$ & $91,3 \%$ & $95,0 \%$ \\
\hline PEnTA & $90,1 \%$ & $93,2 \%$ & $92,7 \%$ & $32,9 \%$ & $90,1 \%$ & $76,2 \%$ \\
\hline PNEUMO 10 & $93,9 \%$ & $95,4 \%$ & $95,7 \%$ & $95,7 \%$ & $93,8 \%$ & $93,8 \%$ \\
\hline VIP & $91,2 \%$ & $94,6 \%$ & $92,5 \%$ & $93,5 \%$ & $92,3 \%$ & $90,1 \%$ \\
\hline $\begin{array}{c}\text { FEBRE } \\
\text { AMARELA }\end{array}$ & & & & & $35,7 \%$ & $77,9 \%$ \\
\hline VTV & $96,9 \%$ & $92,8 \%$ & $97,4 \%$ & $97,4 \%$ & $96,1 \%$ & $96,7 \%$ \\
\hline HEPATITE A & $83,9 \%$ & $107,9 \%$ & $80,2 \%$ & $89,7 \%$ & $91,4 \%$ & $97,0 \%$ \\
\hline
\end{tabular}

*2019 - danos do ano

Fonte: SMS/Núcleo M\&A/Esaude/Cobertura vacinal menores de 1 ano e Cobertura Vacinal de 01 ano em 07/01/2020 outros serviços - SIPNI/Relatório Vacinados por vacina em 10/01/2020.

A hesitação vacinal refere-se ao atraso em aceitar ou recusar as vacinas, apesar da disponibilidade de serviços de vacinação. É um problema mundial complexo, contexto-específico que varia ao longo do tempo, local e tipos de vacinas ${ }^{12}$. Devido à natureza complexa da hesitação vacinal, não existe estratégia única de intervenção que possa atingir a todas as instâncias relacionadas à hesitação vacinal ${ }^{13}$.

As legislações asseguram o direito à saúde como fundamental do ser humano, no Estatuto da Criança e do Adolescente (Lei Federal n. 8.069, de 13 de julho de 1990), no 
art. 14., parágrafo primeiro explicita "É obrigatória a vacinação das crianças nos casos recomendados pelas autoridades sanitárias"14.

Enquanto o artigo 24 da Convenção sobre os Direitos da Criança descreve que:

Os Estados Partes reconhecem o direito da criança de gozar do melhor padrão possível de saúde e dos serviços destinados ao tratamento das doenças e à recuperação da saúde. Os Estados Partes envidarão esforços no sentido de assegurar que nenhuma criança se veja privada de seu direito de usufruir desses serviços sanitários ${ }^{15}$.

Segundo o Ministério da Saúde, não há dados sobre a taxa de recusa vacinal no país. Mas de acordo com o Departamento de Informática do Sistema Único de Saúde do Brasil (Datasus), as oito vacinas obrigatórias até o primeiro ano de vida estão com cobertura abaixo do recomendado pela OMS, que é $90 \%$ a $95 \%{ }^{16}$. De acordo com a UNICEF, se um número suficiente de pessoas em uma determinada comunidade for imunizado contra uma determinada doença, alcança-se algo chamado imunidade coletiva ou "efeito rebanho". Quando isso acontece, as doenças não podem se espalhar facilmente de pessoa para pessoa porque a maioria das pessoas está imune. Isso proporciona uma camada de proteção contra as doenças, mesmo para aqueles que não podem ser vacinados. A imunidade coletiva ou "efeito rebanho" também previne surtos ao dificultar a disseminação da doença. A doença se tornará cada vez mais rara, às vezes desaparecendo completamente da comunidade ${ }^{17}$.

O interesse social pela proteção coletiva é considerado mais importante que a própria imunidade individual, uma vez que quanto mais indivíduos forem vacinados, maior será a imunidade coletiva ${ }^{18}$. Levantada a necessidade de uma definição prática de hesitação vacinal para garantir que Coordenadores de programas de imunização, formuladores de políticas, profissionais e pesquisadores usariam um termo padrão para cobrir a ampla gama de fatores que causam baixa aceitação vacinal. O Grupo de Trabalho concluiu que a hesitação é um fenômeno comportamental que é vacina e contexto específico. É medido em relação à expectativa de atingir uma determinada meta de cobertura vacinal, ditada pelos serviços de imunização ${ }^{12}$.

A queda das coberturas vacinais no Brasil desde 2016 levanta a questão da hesitação vacinal como uma das principais preocupações das autoridades sanitárias. Este fenômeno deve ser entendido como uma continuidade entre aqueles que aceitam e aqueles que recusam indubitavelmente todas as vacinas, ou seja, indivíduos hesitantes situam-se entre esses dois extremos. Eles constituem grupos heterogêneos, nos quais alguns aceitam apenas algumas vacinas e outros atrasam propositalmente, não aceitando o esquema vacinal recomendado. Em proporção menor, há aqueles que recusam apenas algumas vacinas e aqueles que ainda têm dúvidas sobre a decisão de vacinar ou não ${ }^{5}$. 
Esse comportamento é influenciado por muitos fatores inter-relacionados, como a confiança, complacência e conveniência, conhecido como modelo dos "3 Cs", proposto pela OMS em 2011. A confiança é sobre a eficácia e segurança das vacinas, o sistema de saúde que as fornece e as motivações dos gestores para recomendá-las. A complacência resulta da baixa percepção de risco de contrair a doença de forma que a vacinação não seria considerada necessária. Por fim, a conveniência considera a disponibilidade física, disposição para pagar, acessibilidade geográfica, capacidade de compreensão e acesso à informação em saúde ${ }^{5}$.

A hesitação vacinal deve ser mais bem compreendida no contexto brasileiro por meio de publicações científicas. Movimentos antivacinas, apesar de antigos, estão se fortalecendo no mundo, com início mais visível em países de alta renda. Entretanto, certamente, o impacto desse sentimento negativo em relação à vacina será mais importante nos países de média e baixa renda, à medida que esses movimentos se fortaleçam. É possível relacionar que associação desses fatores, hesitação vacinal e movimentos antivacina, têm provocada a baixa cobertura vacinal, nesse sentido é imprescindível que gestores, pesquisadores e a população brasileira se mobilizem para proteger o exitoso programa de imunizações 5 .

Entendendo que a saúde é um direito fundamental, o acesso à vacina, de forma universal, deve ser responsabilidade do Estado, incluindo estratégias de que promovam esclarecimento e empoderamento da população sobre a importância das vacinas.

Contudo, no âmbito da legislação a hesitação vacinal e confiança devem ser permanentemente trabalhadas na comunidade visando assegurar à criança e adolescente "direito a proteção à vida e à saúde, mediante a efetivação de políticas sociais públicas que permitam o nascimento e o desenvolvimento sadio e harmonioso, em condições dignas de existência", artigo $7^{\circ}$ do Estatuto da Criança e do Adolescente, isto completa o artigo 14 que obriga a vacinar, e o artigo $7^{\circ}$ justiça que é responsabilidade compartilhada entre estado e família.

O subitem $\mathrm{f}$ do Artigo 24, Convenção sobre os Direitos da Criança ainda consubstancia esta afirmação, que cabe aos Estados membros da Convenção "desenvolver a assistência médica preventiva, a orientação aos pais e a educação e serviços de planejamento familiar"15.

Porém, cabe ressaltar que as famílias, no contexto da lei da brasileira, especificamente no Estatuto da Criança e do Adolescente (Lei Federal n. 8.069, de 13 de julho de 1990), aponta no artigo $4^{\circ}$ que

É dever da família, da comunidade, da sociedade em geral e do poder público assegurar, com absoluta prioridade, a efetivação dos direitos referentes à vida, à saúde, à alimentação, à educação, ao esporte, ao lazer, à profissionalização, à cultura, à dignidade, ao respeito, à liberdade e à convivência familiar e comunitária ${ }^{14}$. 
Pesquisadores do Strategic Advisory Group of Experts em Imunização da OMS identificaram, descreveram e avaliaram a eficácia potencial de estratégias para responder a questões de hesitação vacinal que foram implementadas e avaliadas em contextos globais diversos. Demonstraram que as intervenções mais eficazes empregaram várias estratégias. As intervenções com os maiores aumentos observados (>25\%) de adesão à vacinação foram aquelas que (não em ordem de importância): (1) diretamente atingiram populações não vacinadas ou sub-vacinadas; (2) visavam aumentar o conhecimento e a conscientização da população sobre a vacinação; (3) melhoraram o acesso a vacinação; (4) foram direcionadas à populações específicas; (5) tornaram a vacinação obrigatória ou impetravam sanção contra a não vacinação; e (6) envolveram líderes religiosos ou outros líderes influentes para promover a vacinação. Os maiores aumentos $(>20 \%)$ em conhecimento, conscientização ou atitudes foram observados em iniciativas de educação, particularmente aquelas que incorporam novos conhecimentos em processos de rotina (por exemplo, procedimentos hospitalares), que tiveram mais sucesso em aumentar o conhecimento e mudar atitudes. Para ambos os resultados, aqueles que adaptaram intervenções a populações específicas e suas preocupações específicas foram mais eficazes ${ }^{19}$.

No Brasil, existem ações na justiça, gerando jurisprudência para outros casos, estas ações visam garantir a vacinação em crianças e adolescentes, mesmo que seus pais ou responsáveis não queiram, neste contexto pode-se exemplificar com a Apelação Cível 10518180076920001/MG do Relator: Dárcio Lopardi Mendes, data de Julgamento: 12/12/2019, data de Publicação: 17/12/2019 tramitou no Tribunal de Justiça de Minas Gerais TJ-MG que aponta

A vacinação consiste não apenas em direito individual, mas em direito coletivo, uma vez que tem por objeto a diminuição, ou até mesmo a erradicação de doenças. A interpretação que se faz é que as normas de regência buscam garantir a saúde do indivíduo e, por consequência, de toda a população, sendo, portanto, algo acima da escolha pessoal, vez que envolve a diminuição da exposição ao risco e ao contágio de determinadas doenças e ainda evita o reaparecimento de doenças consideradas erradicas. Em consideração Ao Princípio Constitucional do Melhor Interesse, não podem os genitores se recursarem a vacinar os filhos quando se busca alcançar o pleno desenvolvimento daqueles, o que, por certo, envolve o direito à saúde em todas as suas formas, incluídas as de prevenção por meio da vacinação. $O$ interesse do menor se sobrepõe a qualquer interesse particular dos genitores. A imposição da imunização não fere o direito à liberdade religiosa, uma vez que não sendo esse absoluto, é passível de ponderação e, assim, não há se falar no direito de escolha dos pais, mas no direito da criança à saúde ${ }^{20}$.

A hesitação vacinal é uma questão complexa e nenhuma estratégia única será capaz de resolvê-la. Uma das maiores desvantagens identificadas nas intervenções é que muitas se baseiam em suposições, ao invés de basearem-se em evidências. Uma avaliação 
dos dados apropriada também é falha. Em uma perspectiva mais positiva, há um crescente corpo de pesquisa sobre os determinantes da hesitação vacinal que podem ajudar a informar e refinar as abordagens atualmente usadas que parecem promissoras, mas que ainda não foi totalmente implementado ou avaliado ${ }^{19}$.

O sucesso das vacinas, erradicando algumas doenças, ou deixando sua ocorrência muito baixa, promoveu um distanciamento entre a necessidade e a preocupação por parte da população, assim as vacinas tornaram-se vítimas do seu próprio êxito, já que as doenças que previnem são desconhecidas pela maioria da população e parecem desnecessárias para muitos pais, que se recusam ou atrasam a vacinação de seus fiIhos. Outra questão abordada pelos autores que reforça esses movimentos é a grande divulgação pela mídia de argumentos antivacinas, o que acaba causando reação pública contra as vacinas ${ }^{21}$. Um dos artigos mais famosos envolveu o ex-pesquisador Andrew Wakefield, que, em 1998 publicara um estudo que correlacionava a vacina do sarampo com o autismo. Até que fosse provado que dados foram falsificados para sugerir esta relação, o efeito danoso sobre a cobertura vacinal em vários países foi notável. As vacinas representam um importante aspecto do cuidado pediátrico. Apesar da hesitação vacinal não ser um assunto novo, um número crescente de pais tem optado por não vacinar seus filhos, representando uma ameaça à saúde pública. Ainda que pesquisas estejam sendo feitas, poucas estratégias baseadas em evidências têm sido adotadas para combater esta questão.

A Política Nacional de Imunização brasileira por sua contribuição e sucesso internacional, traz consigo legados muitas vezes desconhecidos do público, devendo, portanto, haver um diálogo midiático com a sociedade ${ }^{22}$.

Para manter o sucesso dos programas de vacinação, é também de fundamental importância identificar os profissionais que hesitam em vacinar, entender as causas de suas hesitações e desenvolver estratégias personalizadas para lidar com isso. Há urgência na elaboração de estratégias mais eficazes de comunicação midiática com a população da importância de uma política pública de vacinação. Como na população em geral, a hesitação vacinal nos profissionais de saúde é altamente específica ao contexto e variará entre vacinas, profissões e ao longo do tempo ${ }^{23}$.

Estudo publicado mostra que a pouca circulação de informação confiável sobre vacinas está sendo parcialmente preenchida por conteúdo antivacinação e desinformação postados e compartilhados nas redes sociais e nos aplicativos de mensagens - e algumas vezes são criados por pessoas que vendem "curas" alternativas junto com o conteúdo antivacinação. A desinformação também parece afetar a percepção em relação à segurança das vacinas entre aqueles que obtêm suas principais informações sobre vacinas nas redes sociais e em aplicativos de mensagens. Esses indivíduos parecem ser mais 
propensos a acreditar nas informações incorretas a que são expostos. Para identificar se os entrevistados viram ou acreditaram em desinformação sobre vacinas, o responsável pelo estudo forneceu uma lista dos mitos mais relatados sobre vacinação ouvidos pelos médicos, de acordo com a SBIm (Sociedade Brasileira de Imunizações). Quase sete em cada dez brasileiros $(67 \%)$ acreditaram em pelo menos uma declaração factualmente imprecisa sobre vacinas - identificando pelo menos uma como um fato verdadeiro ${ }^{9}$.

Como táticas para combater as "fake news" sobre vacinas, o estudo sugere que as autoridades sanitárias revejam as estratégias de comunicação, buscando canais capazes de ampliar a disseminação das informações de forma mais efetiva. Para combater a desinformação sobre vacinas e os posicionamentos antivacinação, cita que são necessárias mais informações disponíveis ao público. Recomenda que deve haver busca ativa por desinformação antivacinas e trabalho junto à comunidade científica para corrigir as "fake news". A análise dos dados indica que a epidemia da desinformação sobre vacinas não é apenas um problema brasileiro. É uma questão global e pode ser tratada com mais eficácia através de uma estratégia global. Organismos internacionais com Organização Mundial da Saúde, Organização Pan Americana de Saúde e a Fundo de Emergência Internacional das Nações Unidas para a Infância - UNICEF, devem estabelecer estratégias de combater as "fake news", em especial na rede mundial de computadores, e redes sociais.

Grande parte do conteúdo espalhado no Brasil é traduzida de informações originalmente publicadas no exterior - em inglês - e, principalmente, nos Estados Unidos. Mais pesquisas precisam ser feitas para avaliar o impacto da desinformação sobre vacinas no Hemisfério Sul, uma vez que parece ser um fenômeno relativamente novo ${ }^{9}$.

A popularização do uso de dispositivos móveis para capturar e compartilhar todos os tipos de eventos que estão testemunhando, e ainda abundância de versões manipuladas desses dados é propagada pelos mesmos canais, das quais devem ser compelidas com agregação de soluções forenses significativas e o design e desenvolvimento de novos métodos para analisar interações entre fontes heterogêneas, visando a prevenção e investigação de crimes, além de combater a proliferação de notícias falsas ${ }^{24}$.

\section{CONCLUSÃO}

O histórico da imunização na população mundial não deixa dúvidas sobre a eficácia e a eficiência das vacinas e a importância de se manter uma cobertura vacinal adequada. No momento em que o mundo enfrenta uma pandemia de uma doença transmissível, que uniu esforços de toda a comunidade científica em busca da produção de uma vacina eficaz e segura contra a COVID-19, parece impossível imaginar movimentos que poderiam contrariar a necessidade da descoberta e da proteção da população por meio 
de um insumo imunobiológico. No entanto, segundo estudos preliminares publicados no site da Fundação Oswaldo Cruz, a mais destacada instituição de ciência e tecnologia em saúde da América Latina, vinculada ao Ministério da Saúde, já demonstram que o dano começa a ser mensurado até mesmo antes da disponibilização de uma vacina para a COVID-19: estudo publicado na Lancet indica que $26 \%$ dos franceses não tomariam a vacina se ela já existisse; trabalho conduzido pela Universidade de Cambridge aponta que $12 \%$ dos cidadãos do Reino Unido não se vacinariam e $18 \%$ tentariam influenciar seus conhecidos a não fazê-lo; e pesquisa da Reuters revela que 1 a cada 4 pessoas nos Estados Unidos - impressionantes $25 \%$ da população - não tem interesse em se vacinar contra a COVID-1925.

Cabe aqui citar outras epidemias com rápidas e mortais disseminações: a varíola, com mais de 300 milhões de mortes estimadas durante todo o século XX; a Gripe Espanhola (1918-1920), que deixou aproximadamente 50 milhões de mortos em todos os continentes e a pandemia mais recente, em 2009, do vírus H1N1. A medida que estas doenças tiveram declínio nas suas incidências, ou até mesmo, foram erradicadas, principalmente devido às estratégias de vacinação em massa, a sensação de segurança na população em não contrair a enfermidade aumentou, e juntamente com ela, notícias e movimentos sem fundamento contra a utilização dos imunobiológicos.

A queda da cobertura vacinal no Brasil pode ser considerada multifatorial, incluindo razões como desinformação, disseminação das "fake news" e despreocupação por doenças com baixa ocorrência. $O$ decréscimo substancial nos últimos anos merece especial atenção no sentido de provocar discussões e estudos que demonstrem a importância da manutenção da cobertura vacinal adequada, possibilitando melhor entendimento deste assunto por parte da população e da comunidade acadêmico-científica.

Avaliando este contexto, e considerando seu impacto nos países, de média e baixa rendas, o fenômeno comportamental da hesitação vacinal torna-se, portanto, um dos desafios a ser enfrentado pelos gestores da Administração Pública, e profissionais da saúde. Primeiramente, no sentido de se levantar dados quantitativos e qualitativos sobre o assunto. Em seguida, na busca e elaboração de planos para intervenções direcionadas de acordo com a vacina, o público-alvo e o contexto envolvido nas causas da hesitação.

E, finalmente, intervir sobre o danoso compartilhamento de "fake news", que acaba avançando numa velocidade maior do que estudos e informações fundamentadas e provenientes de fontes confiáveis. A revisão das estratégias de comunicação de informações, incluindo o reforço sobre a segurança e eficácia dos imunobiológicos, bem como a vigilância das coberturas vacinais são essenciais e fundamentais no sentido de coibir quaisquer movimentos que possam refletir negativamente nestes números, e consequentemente ferir o direito a proteção à vida e à saúde da população. 
Novos estudos devem ser realizados sobre a cobertura que alcançaremos na vacinação para COVID-19, houve um grande esforço dos cientistas, das instâncias regulatórias e da mídia democratizando as informações técnicas sobre eficiência, segurança e eficácia das vacinas, podendo ter sido uma grande "vacina" contra a hesitação vacinal e o avanço dos movimentos antivacinas.

\section{REFERÊNCIAS}

1. Aps LRMM, Piantola MAF, Pereira SA, Castro JT, Santos FAO, Ferreira LCS. Eventos adversos de vacinas e as consequências da não vacinação: uma análise crítica. Rev. Saúde Pública [Internet]. 2018 [acesso em 2020 out 13]; 52: 40. Disponível em: http://dx.doi.org/10.11606/s1518-8787.2018052000384.

2. Hochman G. Vacinação, varíola e uma cultura da imunização no Brasil. Ciênc. saúde coletiva [Internet]. 2011 [acesso em 2020 out 13]; 16(2): 375-386. Disponível em: https://doi.org/10.1590/S141381232011000200002 .

3. Brasil. Ministério da Saúde. Secretaria de Vigilância em Saúde. Saúde Brasil 2012: uma análise da situação de saúde e dos 40 anos do Programa Nacional de Imunizações.: volume único [Internet]. 1. ed. Brasília: Ministério da Saúde; 2013 [acesso em 2019 dez 12]. 538 p. Disponível em: http://bvsms.saude. gov.br/bvs/publicacoes/saude_brasil_2012_analise_situacao_saude.pdf

4. Ferreira ABH. Dicionário Aurelio da Língua Portuguesa. 5. ed. Curitiba: Positivo; 2010.

5. Sato APS. Qual a importância da hesitação vacinal na queda das coberturas vacinais no Brasil?. Revista de Saúde Pública [Internet]. 2018 [acesso em 2019 abr 22];52(96):1-9. Disponível em: http://www.rsp.fsp. usp.br/artigo/qual-a-importancia-da-hesitacao-vacinal-na-queda-das-coberturas-vacinais-no- brasil/.

6. Dubé E, Vivion M, MacDonald NE. Vaccine hesitancy, vaccine refusal and the anti-vaccine movement: influence, impact and implications, Expert Review of Vaccines, 14:1, 99-117. Disponível em: https://doi.org/ $10.1586 / 14760584.2015 .964212$

7. World Health Organization. Ten threats to global health in 2019 [internet]. [acesso em 2019 mai 18]. Disponível em: https://www.who.int/emergencies/ten-threats-to-global-health-in-2019

8. Brasil. Ministério da Saúde. Secretaria de Vigilância em Saúde. Manual de Normas e Procedimentos para Vacinação: volume único [Internet]. 1. ed. Brasília: Ministério da Saúde; 2014 [acesso em 2019 dez 12]. 178 p. Disponível em: https://portalarquivos2.saude.gov.br/images/pdf/2019/junho/25/guia-vigilancia-saude-volume-unico-3ed.pdf

9. AVAAZ, SBim. As Fake News estão nos deixando doentes? [Internet]. 2019. [acesso em 2019 nov 24]. Disponível em: https://sbim.org.br/images/files/po-avaaz-relatorio-antivacina.pdf

10. Brasil. Ministério da Saúde. A queda da Imunização no Brasil [internet]. 2017. [acesso em 2019 nov 24]. Disponível em: https://www.conass.org.br/consensus/queda-da-imunizacao-brasil/

11. Fundação Oswaldo Cruz. E agora Zé: Baixa cobertura das principais vacinas compromete programa brasileiro de imunizações, reconhecido internacionalmente [Internet]. 2019. [acesso em 2019 nov 24]. Disponível em: https://radis.ensp.fiocruz.br/index.php/home/reportagem/e-agora-ze

12. MacDonald NE; SAGE Working Group on Vaccine Hesitancy. Vaccine hesitancy: Definition, scope and determinants. Vaccine. 2015;14:33(34):4161-4. Disponível em: https://doi.org/10.1016/j.vaccine.2015.04.036

13. Dubé E, Gagnon D, MacDonald NE; SAGE Working Group on Vaccine Hesitancy. Strategies intended to address vaccine hesitancy: Review of published reviews. Vaccine. 2015 14;33(34):4191-203. Disponível em: https://doi.org/ 10.1016/j.vaccine.2015.04.041

14. Brasil. Presidência da República. Lei Federal n. 8.069, de 13 de julho de 1990: Dispõe sobre o Estatuto da Criança e do Adolescente e dá outras providências [internet]. 1990. [acesso em 2020 jun 04]. Disponível em: http://www.planalto.gov.br/ccivil_03/leis//8069.htm 
15. Brasil. Presidência da República. Decreto n. 99.710, de 21 de novembro de 1990: Promulga a Convenção sobre os Direitos da Criança [internet]. 1990. [acesso em 2020 jun 04]. Disponível em: http://www. planalto.gov.br/ccivil_03/decreto/1990-1994/d99710.htm

16. Agência Brasil. Campanhas buscam elevar cobertura vacinal no país [internet]. 2019. [acesso em 2020 abr 25]. Disponível em: https://agenciabrasil.ebc.com.br/saude/noticia/2019-04/campanhas-buscam-elevar-cobertura-vacinal-no-pais

17. UNICEF. VACINAS [internet]. [acesso em 2020 abr 25]. Disponível em: https://www.unicef.org/brazil/ vacinas-perguntas-e-respostas

18. Lessa SC, Schramm FR. Proteção individual versus proteção coletiva: análise bioética do programa nacional de vacinação infantil em massa. Ciênc. saúde coletiva [Internet]. 2015 [acesso em 2020 out 14]; 20(1):115-124. Disponível em: http://dx.doi.org/10.1590/1413-81232014201.14882013.

19. Jarrett C, Wilson R, O'Leary M, Eckersberger E, Larson HJ, SAGE Working Group on Vaccine Hesitancy. Strategies for addressing vaccine hesitancy: A systematic review. Vaccine [Internet]. 2018:14;33(34):418090. Disponível em: https://pubmed.ncbi.nlm.nih.gov/25896377/

20. TRIBUNALDE JUSTIÇADE MINAS GERAIS (TJ-MG). Resumo daApelação Cível 10518180076920001/ MG [internet]. 2019. [acesso em 2020 jul 06]. Disponível em: https://tj-mg.jusbrasil.com.br/jurisprudencia/793583412/apelacao-civel-ac-10518180076920001-mg.

21. McClure CC, Cataldi JR, O'Leary ST. Vaccine Hesitancy: Where We Are and Where We Are Going. Clin Ther. [Internet] 2017;39(8):1550-1562. Disponível em: https://pubmed.ncbi.nlm.nih.gov/28774498/

22. Temporão JG. O Programa Nacional de Imunizações (PNI): origens e desenvolvimento. Hist. cienc. saude-Manguinhos [Internet]. 2003 [acesso em 2020 out 14]; 10 (Suppl 2): 601-617. Disponível em: https:// doi.org/10.1590/S0104-59702003000500008.

23. Dubé E. Addressing vaccine hesitancy: the crucial role of healthcare providers. Clinical Microbiology and Infection [Internet]. 2017;23(5):279-280. Disponível em: https://pubmed.ncbi.nlm.nih.gov/27851999/

24. Ferreira A, Carvalho T, Andaló F, Rocha A. Counteracting the contemporaneous proliferation of digital forgeries and fake news. An. Acad. Bras. Ciênc. [Internet]. 2019 [acesso em 2020 oct 14]; 91(Suppl1):e20180149. Disponível em: https://doi.org/10.1590/0001-3765201820180149.

25. Fundação Oswaldo Cruz. A pandemia da desinformação [internet]. 2020. [acesso 2020 jul 22]. Disponível em: https://www.bio.fiocruz.br/index.php/br/noticias/1883-a-pandemia-da-desinformacao?fbclid=IwAR1Vv004KGI2ghct_hrLMn-nHsWz4_P_no7n9akBcG5y85mIRVovrtgrRbQ 